\title{
Characterization and Morphology of Modified Multi- Walled Carbon Nanotubes Filled Thermoplastic Natural Rubber (TPNR) Composite
}

\author{
Mou'ad A. Tarawneh and Sahrim Hj. Ahmad \\ Additional information is available at the end of the chapter \\ http://dx.doi.org/10.5772/50726
}

\section{Introduction}

Carbon nanotubes describes a specific topic within solid-state physics, but is also of interest in other sciences like chemistry or biology. Actually the topic has floating boundaries, because we are at the molecule level. In the recent years carbon nanotubes have become more and more popular to the scientists. Initially, it was the spectacularly electronic properties, that were the basis for the great interest, but eventually other remarkable properties were also discovered.

The first CNTs were prepared by M. Endo in 1978, as part of his PhD studies at the University of Orleans in France. Although he produced very small diameter filaments (about $7 \mathrm{~nm}$ ) using a vapour-growth technique, these fibers were not recognized as nanotubes and were not studied systematically. It was only after the discovery of fullerenes, C60, in 1985 that researchers started to explore carbon structures further. In 1991, when the Japanese electron microscopist Sumio Iijima [1] observed CNTs, the field really started to advance. He was studying the material deposited on the cathode during the arc-evaporation synthesis of fullerenes and came across CNTs. A short time later, Thomas Ebbesen and Pulickel Ajayan, from Iijima's lab, showed how nanotubes could be produced in bulk quantities by varying the arc-evaporation conditions. However, the standard arc-evaporation method only produced only multiwall nanotubes. After some research, it was found that the addition of metals such as cobalt to the graphite electrodes resulted in extremely fine single wall nanotubes.

The synthesis in 1993 of single-walled carbon nanotubes (SWNTs) was a major event in the development of CNTs. Although the discovery of CNTs was an accidental event, it opened the way for a flourishing research into the properties of CNTs in labs all over the world, with many scientists demonstrating promising physical, chemical, structural, and optical properties of CNTs. 
CNTs exhibit a great range of remarkable properties, including unique mechanical and electrical characteristics. These remarkable high modulus and stiffness properties have led to the use of CNTs to reinforce polymers in the past few years. Both theoretical (e.g. molecular structural mechanics and tight-binding molecular dynamics) and experimental studies have shown SWCNTs to have extremely high elastic modulus ( $\sim 1 \mathrm{TPa})$ [2-3]. The tensile strength of SWCNTs estimated from molecular dynamics simulation is $\approx 150 \mathrm{MPa}$ [4]. The experimental measurement of $150 \mathrm{MPa}$ was found for the break strength of multi-walled carbon nanotubes (MWCNTs) [5].

The remarkable properties of CNTs offer the potential for improvement of the mechanical properties of polymers at very low concentrations. In practice, MWCNTs are preferred over SWCNTs as the reinforcing fillers for polymers due to their lower production cost. However, slippage between the shells of MWCNTs would undermine the capability of the fillers to bear the external applied load.

Mixed 1 wt.\% MWCNTs with polystyrene (PS) in toluene via ultrasonication, achieved about $36-42 \%$ increase in the elastic modulus and a $25 \%$ increase in the tensile strength of the PS-MWCNT film compared to pure PS [6]. They found that nanotube fracture and pullout are responsible for the failure of the composite. The fracture of MWCNTs in a PS matrix implies that certain load transfer from the PS to the nanotubes has taken place. However, the pullout of MWCNTs from the PS matrix indicating that the PS-nanotube interfacial strength is not strong enough to resist debonding of the fillers from the matrix. It is considered that some physical interactions exist at the PS-MWCNT interface, thereby enabling load transfer from the matrix to the fillers.

The additions of $0.25-0.75 \mathrm{wt}$ \% SWCNTs to polypropylene (PP) considerably its tensile strength and stiffness as well as storage modulus. The elongation at break reduces from 493 (PP) to $410 \%$ with the addition of $0.75 \mathrm{wt}$. \% filler, corresponding to $-17 \%$ reduction in ductility. At $1 \mathrm{wt} . \% \mathrm{SWNT}$, both stiffness and strength are significantly reduced due to the formation of aggregates [7].

The morphology and mechanical properties of the melt-compounded polyamide 6 (PA6)MWNT nanocomposites were studied by [8]. The MWCNTs were purified by dissolving the catalyst in hydrochloric acid followed by refluxing in 2.6 Mnitric acids to increasing the carboxylic and hydroxyl groups. It was also found that with the addition of only $1 \mathrm{wt} . \%$ MWCNTs, the tensile modulus and the tensile strength are greatly improved by $\approx 115$ and $120 \%$, respectively compared to neat PA6. The tensile ductility drops slightly from 150 to $125 \%$. They attributed the improvements of these mechanical properties to a better dispersion of MWCNTs in PA6 matrix, and to a strong interfacial adhesion between the nanofillers and PA6 matrix which leads to favorable stress transfer across the polymer to the MWCNTs.

The influence of SWNT and carbon nanofiber additions on the mechanical performances of silicone rubber was reported by [9]. They reported that SWCNTs are effective reinforcements for silicone rubber due to their large aspect ratio and low density. The initial modulus (measured by fitting a straight line to the data below 10\% strain) tends to increase almost linearly with increasing filler content. The effect of SWCNT and carbon fiber additions on the tensile ductility of silicone rubber is shown that the strain to failure drops from 325 to $275 \%$ upon loading with $1 \mathrm{wt}$ \% SWCNTs, corresponding to $\approx 15 \%$ reduction. 
The carbon nanotube additions to polyurethane (PU) improve the mechanical properties such as increased modulus and yield stress, without loss of the ability to stretch the elastomer above $1000 \%$ before final failure; the addition of CNTs increases the modulus and strength of PU without degrading deformabilty. The elongation at break decreases very slightly with CNT loading up to 17 wt.\%. At this filler loading, the nanocomposite still maintains a very high value of elongation at rupture, i.e. 1200\% [10].

Theoretical prediction showed an extremely high thermal conductivity $(6000 \mathrm{~W} / \mathrm{mK})$ of an isolated SWCNTs [11]. High thermal conductivity of the CNTs may provide the solution of thermal management for the advanced electronic devices with narrow line width. Revealed the thermal conductivity of epoxy-based composites reinforced with $1.0 \mathrm{wt}$ \% SWCNTs increased over $125 \%$ reaching a value of $\sim 0.5 \mathrm{~W} / \mathrm{mK}$ [12]. The variation of thermal conductivity with the values of 35 and $2.3 \mathrm{~W} / \mathrm{mK}$ for a densepacked mat and a sintered sample, respectively [13]. High thermal conductivity of 42 and $\sim 18 \mathrm{~W} / \mathrm{mK}$ of the aligned and the random bucky paper mats, respectively. However, the thermal conductivity drops significantly by almost an order of magnitude when the aligned bucky paper mats were loaded with epoxy, the volume fraction of the aligned bucky paper composites is about $50 \%[14]$.

Developed an infiltration method to produce CNTs/epoxy composites and showed a $220 \%$ increase in thermal conductivity $(\sim 0.61 \mathrm{~W} / \mathrm{mK})$ at $2.3 \mathrm{wt} . \%$ SWCNT loading, and they found that the electrical resistance between SWCNT-polymer is more severe than that of SWCNTSWCNT [15]. Prepared SWCNT and MWCNT films and reported the thermal conductivity of 1.64 and $1.51 \mathrm{~W} / \mathrm{mK}$, respectively; they concluded that the intra-tube spacing affects the thermal transfer more significantly than that of the nanotubes themselves [16].

The thermal conductivities of composites reinforced with $1.0 \mathrm{wt} . \%$ SWCNTs and $4.0 \mathrm{wt} . \%$ MWCNTs are $2.43 \mathrm{~W} / \mathrm{mK}$ and $3.44 \mathrm{~W} / \mathrm{mK}$, respectively. Composites reinforced with the unpurified CNTs have higher thermal conductivity than that of the purified CNTs reinforced composite. This is attributed to the generation of defects on the CNT surface during acid treatment. Moreover, due to longer phonon propagation length, it is found that thermal conductivity increases with temperatures over the range from 25 to $55^{\circ} \mathrm{C}$ for both SWCNTs/ Poly (methyl methacrylate) PMMA and MWCNTs/PMMA composites. However, the thermal conductivities of CNT films decrease with increasing temperature, which results from phonon scattering during transfer due to the presence of defects coupled with smaller phonon mean free path at higher temperature [17].

The differences in the composite manufacturing methods, powder-(MWCNTs and ball milled SWCNTs) or liquid- (chemically treated SWCNTs) based approach, can not account for the differences in the properties, since both methods were used for the SWNT-composites and resulted in similar thermal behaviour [18]. Thus, they concluded that in this case, there must be a very large interface resistance to the heat flow associated with poor phonon coupling between the stiff nanotubes and the (relatively) soft polymer matrix. In addition it is possible that the phonon vibrations in the SWCNTs are dampened by the matrix interaction, while in the MWCNTs the phonons can be carried in the inner walls without hindrance. 
The precise sectioning of CNTs provides an effective way to shorten carbon nanotubes with controlled length and minimum sidewall damage [19]. For shortened nanotubes they found that they are easily dispersed into polymer matrices, which effectively improved the percolation. The minimum CNT sidewall damage and improved percolation in short SWCNT composites led to an obvious improvement of thermal conductivity. Hence, their research suggests an effective way to improve dispersion of CNTs into polymer matrices and also retain the perfect electronic structure of the CNTs, resulting in desired functional materials.

Accurate measurement of the thermal conductivity of composites and nanocomposites can be done using the transient hot-wire technique which is capable of measuring the thermal conductivity of solid materials in an absolute way. The enhancement in the thermal conductivity was measured as $27 \%$ in relation to the thermal conductivity of the epoxy-resin polymer, which is satisfactory taking into account the low volume fraction (28\%) of the glass fibres used in the composite [20]. They reported that when $2 \%$ by weight C-MWNT were mixed with the epoxy-resin, the enhancement of thermal conductivity was $9 \%$ while using both glass fibres and C-MWNT the enhancement was $48 \%$.

For sufficient enhancement of most of the nanocomposites' properties, the dispersion of the CNTs should be very fine in the polymer matrix, which means that the surface of interaction between the filler and the matrix should be optimised. However, this is difficult to achieve since their long length results in them becoming entangled. Moreover, their very large surface-to-volume ratio and strong van der Waals interactions keep them tied together, which in most cases leads to the formation of large agglomerates in polymer matrices. The interfacial adhesion between CNTs and the polymeric matrix is also crucial. In order to increase the interfacial adhesion between the polymer and the CNTs various routes of surface modification of the nanotubes have been considered. One is non-covalent functionalisation of molecules and the other is covalent functionalisation from the walls of the nanotubes. Noncovalent functionalisation is based on weak Van der Waals forces [21]. The advantage of non-covalent functionalisation is that the perfect structure of the nanotubes is not altered while the covalent attachment can greatly improve the load transfer to the matrix; however, it usually introduces structural defects on the nanotubes' surface.

Although both probe style and bath style ultrasonic systems can be used for dispersing CNTs, it is widely believed that the probe style ultrasonic systems work better for dispersing CNTs [22]. It is also widely known that adding a dispersing reagent (surfactant) into the solution will accelerate the dispersion effect.

The most common procedure used for covalent attachment of reactive groups is the treatment with inorganic acids. Usually the nanotubes are refluxed with a nitric acid solution or a mixture of nitric and sulfuric acid, sometimes concurrently with the application of high power sonication [23]. These oxidative treatments usually result in shortening of the CNTs' length and formation of surface reactive groups, such as hydroxyl, carbonyl and mainly carboxylic acid. Oxidation of the nanotubes starts at the tips and gradually moves towards the central part of the tube and the layers are removed successively [24]. 
The synthesized carbon nanotubes usually exist as agglomerates of the size of several hundred micrometers [25]. Such entanglements make it difficult to disperse nanotubes uniformly in a polymer matrix. To overcome the dispersion problem, it is necessary to tailor the chemical nature of the nanotube surface. One of the most straightforward methods for nanotube dispersion is direct mixing; however, it does not always yield a homogeneous distribution of nanotubes because of the lack of compatibility between the MWCNTs and polymer matrix. Solution processing has been a commonly used method in fabrication of the welldispersed carbon nanotube composites. However, it is hard to achieve homogeneous dispersion of nanotubes in a polymer matrix because carbon nanotubes are insoluble and bundled.

Chemical functionalization of the MWCNTs surface increases the interfacial interaction between MWCNTs and the polymer matrix. This enhances the adhesion of the MWCNTs in various organic solvents and polymers, reduces the tendency to agglomerate, and improves dispersion. The improved interactions between MWCNTs and the polymer matrix govern the load-transfer from the polymer to the nanotubes and, hence, increase the reinforcement efficiency. Attachment of oxygen containing functional groups (i.e., carboxyl groups, carbonyl groups, hydroxyl groups, etc.) on the surface of the MWCNTs could be achieved by applying several chemical treatments.

The chemically functionalized MWCNTs can be easily mixed with the polymer matrix. Acid treatment of the nanotube is an especially well-known technique to remove catalytic impurities, generate functional groups on open ends or sidewalls of nanotubes, and facilitate good dispersion of MWCNTs in polymeric solutions or melts.

The emergence of thermoplastic elastomers (TPEs) is one of the most important developments in the area of polymer science and technology. TPEs are a new class of material that combines the properties of vulcanized rubber with the ease of processability of thermoplastics [26]. Thermoplastic elastomers can be prepared by blending thermoplastic and elastomers at a high shear rate. Thermoplastics, for example, polypropylene (PP), polyethylene (PE) and polystyrene (PS), and elastomers, such as ethylene propylene diene monomer (EPDM), natural rubber (NR) and butyl rubber (BR), are among the materials used in thermoplastic elastomer blends.

Blends of natural rubber (NR) and polypropylene (PP) have been widely reported by previous researchers [26]. According to them, polypropylene is the best choice for blending with natural rubber due to its high softening temperature $\left(150^{\circ} \mathrm{C}\right)$ and low glass transition temperature $\left(-60^{\circ} \mathrm{C}\right.$, is $\mathrm{Tg}$ for NR), which makes it versatile in a wide range of temperatures. Even though NR and PP are immiscible, their chemical structure is nearly the same. Thus, stable dispersion of NR and PP is possible. Incompatibility between NR and PP can be overcome by the introduction of a compatibiliser that can induce interactions during blending. Compatibility is important as it may affect the morphology, mechanical and thermal properties of the blends. Among the commonly used compatibilisers are dicumyl peroxide (DCP), m-phenylene bismaleimide (HVA-2) and liquid natural rubber (LNR). Apart from compatibility, mixing torque and curing are interrelated in determining the homogeneity of the TPNR blend. 
Mechanical blending of PP and NR with the addition of LNR as a compatibiliser has been reported to be optimal at a temperature of $175-185^{\circ} \mathrm{C}$ and a rotor speed of 30-60rpm. The percentage of LNR used depends on the ratio of NR to PP. For a NR:PP ratio of 30:70 the best physical properties are obtained at 10\% LNR [27]. The compatibiliser helps to induce the interaction between the rubber and plastic interphase and thereby increases the homogeneity of the blend.

MWCNTs/TPNR composites with different amounts of MWCNT were prepared and their thermal properties have been investigated by [28]. The higher thermal conductivity was achieved in the samples with 1 and $3 \mathrm{wt} \%$ of MWCNTs compared to the pristine TPNR. Any sample with MWCNTs content higher than $3 w t \%$ caused the conductivity to decrease. In addition, the improvement of thermal diffusivity and specific heat was also achieved at the same percentage. DMA confirmed that the glass transition temperature (Tg) increased with the increase in the amount of MWCNTs.

The tensile strength, tensile modulus, and also the impact strength of TPNR/MWCNTs are improved significantly while sacrificing high elongation at break by incorporating MWCNTs. The reinforcing effect of MWCNTs was also confirmed by DMA where the addition of nanotubes has increased the storage modulus, the loss modulus, and also the glass transition temperature (Tg). Homogeneous dispersion of MWCNTs throughout the TPNR matrix and strong interfacial adhesion between MWCNTs and matrix as confirmed by SEM images are proposed to be responsible for the significant mechanical enhancement [29].

The reinforcing effect of two types of MWCNTs has also confirmed by dynamic mechanical analysis where the addition of nanotubes have increased in the storage modulus E', and the loss modulus E", in the addition the glass transition temperature (Tg) increased with an increase in the amount of MWCNTs. The addition of MWCNTs in the TPNR matrix improved the mechanical properties. The tensile strength and elongation at break of MWCNTs 1 increased by $23 \%$, and $29 \%$, respectively. The Young's modulus had increased by increasing the content of MWCNTs. For MWCNTs 2 the optimum result of tensile strength and Young's modulus was recorded at 3\% which increased 39\%, and 30\%, respectively. The laser flash technique was used to measure the thermal conductivity, thermal diffusivity and specific heat, from the results obtained. The high thermal conductivity was achieved at $1 \mathrm{wt} \%$ and $3 \mathrm{wt} \%$ of MWCNTs compared with TPNR after $3 \mathrm{wt} \%$ it decreased, also the improvement of thermal diffusivity and specific heat was achieved at the same percentage. The MWCNTs 1 and 2/TPNR nanocomposites were fabricated and the tensile and properties were measured [30].

In this chapter, the effect of multi-walled carbon nanotubes with and without acid treatment on the properties of thermoplastic natural rubber (TPNR) was investigated. Two types of MWCNTs were introduced into TPNR, which are untreated multi-walled carbon nanotubes (UTMWCNTs) (without acid treatment) and treated multi-walled carbon nanotubes (TMWCNTs) (with acid treatment). Using this method, MWCNTs are dispersed homogeneously in the TPNR matrix in an attempt to increase the properties of these nanocomposites. The effect of MWCNTs on the mechanical and thermal properties of TPNR nanocomposites is reported in this chapter. 


\section{Experiment Details}

Polypropylene, with a density of $0.905 \mathrm{~g} \mathrm{~cm}-3$, was supplied by Propilinas (M) Sdn. Bhd, natural rubber was supplied by Guthrie (M) Sdn. Bhd, and polypropylene (PP) with a density of $0.905 \mathrm{~g} / \mathrm{cm} 3$ was supplied by Polipropilinas (M) Sdn. Bhd were used in this research. Maleic anhydride-grafted-polypropylene (MAPP) with a density of $0.95 \mathrm{~g} / \mathrm{cm} 3$ was supplied from Aldrich Chemical Co., USA. Liquid natural rubber (LNR) was prepared by the photochemical degradation technique.

A Multi-walled carbon nanotubes (MWCNTs) were provided by Arkema (GraphistrengthTM C100). Table 1 shows the properties of multi-walled carbon nanotubes (MWCNTs).

\begin{tabular}{|c|c|c|c|c|}
\hline MWCNTs & Purity & Length & Diameter & Manufactured \\
\hline & & & & Catalytic Chemical Vapour \\
\hline MWCNTS & "/90\% & $0.1-10 \mu \mathrm{m}$ & $10-15 \mathrm{~nm}$. & $\begin{array}{c}\text { Deposition } \\
\text { (CVD) }\end{array}$ \\
\hline
\end{tabular}

Table 1. Properties of multi-walled carbon nanotubes (MWCNTs).

\subsection{Preparation of TPNR-Multi-Walled Carbon Nanotubes (MWCNTs) Composite}

Mixing was performed by an internal mixer (Haake Rheomix 600P). The mixing temperature was $180^{\circ} \mathrm{C}$, with a rotor speed of $100 \mathrm{rpm}$ and $13 \mathrm{~min}$ mixing time. The indirect technique (IDT) was used to prepare nanocomposites, this involved mixing the MWCNTs with LNR separately, before it was melt blended with PP and NR in the internal mixer. TPNR nanocomposits were prepared by melt blending of PP, NR and LNR with MWCNTs in a ratio of $70 \mathrm{wt} \% \mathrm{PP}, 20 \mathrm{wt} \% \mathrm{NR}$ and $10 \mathrm{wt} \%$ LNR as a compatibiliser and 1,3,5 and $7 \%$ MWCNTs.

\subsection{Acid Treatment of MWCNTs}

Two types of MWCNTs were introduced to the TPNR which is untreated MWCNTs (MWCNTs 1) and treated MWCNTs (MWCNTs 2), MWCNTs 2 were treated by immersing neat MWCNTs in a mixture of nitric and sulfuric acid with a molar ratio of 1:3, respectively. In a typical experiment, $1 \mathrm{~g}$ of raw MWCNTs was added to $40 \mathrm{ml}$ of the acid mixture. Then, the oxidation reaction was carried out in a two-necked, round-bottomed glass flask equipped with reflux condenser, magnetic stirrer and thermometer. The reaction was carried out for 3 hours at $140^{\circ} \mathrm{C}$. After that, this mixture was washed with distilled water on a sintered glass filter until the $\mathrm{pH}$ value was around 7 , and was dried in a vacuum oven at $70^{\circ} \mathrm{C}$ for 24hours [31]. 


\subsection{Characterizations}

Fourier transform infrared (FTIR) spectroscopy analysis was carried out on the Perkin Elmer spectrum V-2000 spectrometer by the potassium bromide (KBr) method for MWCNTs. The samples were scanned between 700 to $4000 \mathrm{~cm}-1$ wave number. Differences in the peaks as well as the new peaks of MWCNTs and MWCNTs after acid treatment were observed to identify any functional groups on the MWCNTs tubes surface.

The tensile properties were tested using a Testometric universal testing machine model M350-10CT with $5 \mathrm{kN}$ load cell according to ASTM 412 standard procedure using test specimens of $1 \mathrm{~mm}$ thickness and a crosshead speed $50 \mathrm{~mm}$ min-1. At least five samples were tested for each composition, and the average value was reported.

The impact test was carried out using a Ray Ran Pendulum Impact System according to ASTM D 256-90b. The velocity and weight of the hammer were $3.5 \mathrm{~m} / \mathrm{s}$ and $0.898 \mathrm{~kg}$, respectively.

Dynamic mechanical analysis for determining the glass transition temperature, storage and loss modulus was carried out using DMA 8000 (PerkinElmer Instrument), operating in single cantilever mode from -100 to $150^{\circ} \mathrm{C}$ at a constant frequency of $1 \mathrm{~Hz}$, with a heating rate of $5^{\circ} \mathrm{C} / \mathrm{min}$. The dimensions of the samples were $30 \times 12.5 \times 3 \mathrm{~mm}$.

The thermal conductivity was measured by a laser flash method. Disk-type samples (12.7 $\mathrm{mm}$ in diameter and $1 \mathrm{~mm}$ in thickness) were set in an electric furnace. Specific heat capacities were measured with a differential scanning calorimeter DSC. Thermal diffusivity $(\lambda$, Wm_1 K_1) was calculated from thermal diffusivity ( $\left.\alpha, \mathrm{m} 2 \mathrm{~s} \_1\right)$, density (Q, g cm_3) and specific heat capacity (Cp, J g_1 K_1) at each temperature using the following:

$$
\lambda=\alpha \cdot \rho . \mathrm{C} .
$$

The reference used for the heat capacity calculation was a $12.7 \mathrm{~mm}$ thick specimen of pyroceram. The reference sample was coated with a thin layer of graphite before the measurement was performed. The thermal conductivity of MWCNTs reinforced TPNR matrix composites of all volume fractions was studied from $30^{\circ} \mathrm{C}$ to $150^{\circ} \mathrm{C}$. The morphology of the MWCNTs and the composite were examined using a scanning electron microscope (Philips $X L$ 30). The samples were coated with a thin layer of gold to avoid electrostatic charging during examination.

\section{Results and Discussion}

\subsection{Fourier-Transform Infrared Spectroscopy}

The method used to functionalize the pristine MWCNTs in this study was the acid treatment method, which is described in section 2.2. Through this process, MWCNTs were oxidized and purified by eliminating impurities such as amorphous carbons, graphite particles, and metal catalysts [32]; the functional group of the surface of the CNTs are as shown in Figure 1. 
The generation of chemical functional groups on MWCNTs was confirmed using Fourier transform infrared spectroscopy (FT-IR) spectra which were recorded between 400 cm_1 and 4000 cm_1.

The FT-IR spectra of pure MWCNTs and the surface treated MWCNTs are shown in Figure. 2 and Figure 3. The characteristic bands due to generated functional groups are observed in the spectrum of each chemically treated MWCNTs. In figure 2 we could not see any band compared with the treated MWCNTs. The acid treated MWCNTs shows new peaks in comparison with the FT-IR spectrum of the untreated MWCNTs, which lack the hydroxyl and carbonyl groups. The peaks around $1580 \mathrm{~cm} \_1$ are assigned to the $\mathrm{O}-\mathrm{H}$ band in $\mathrm{C}-\mathrm{OH}$, and the peaks at $674 \mathrm{~cm}-1$ are assigned to $\mathrm{COOH}$, as shown in Figure 3. This demonstrates that hydroxyl and carbonyl groups have been introduced on the nanotube surface [33].

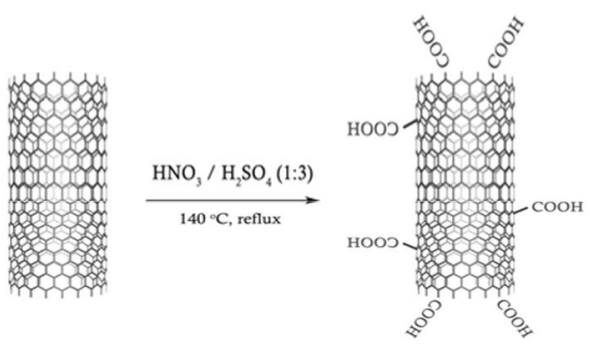

Figure 1. Example of chemical functionalization of carbon nanotubes.

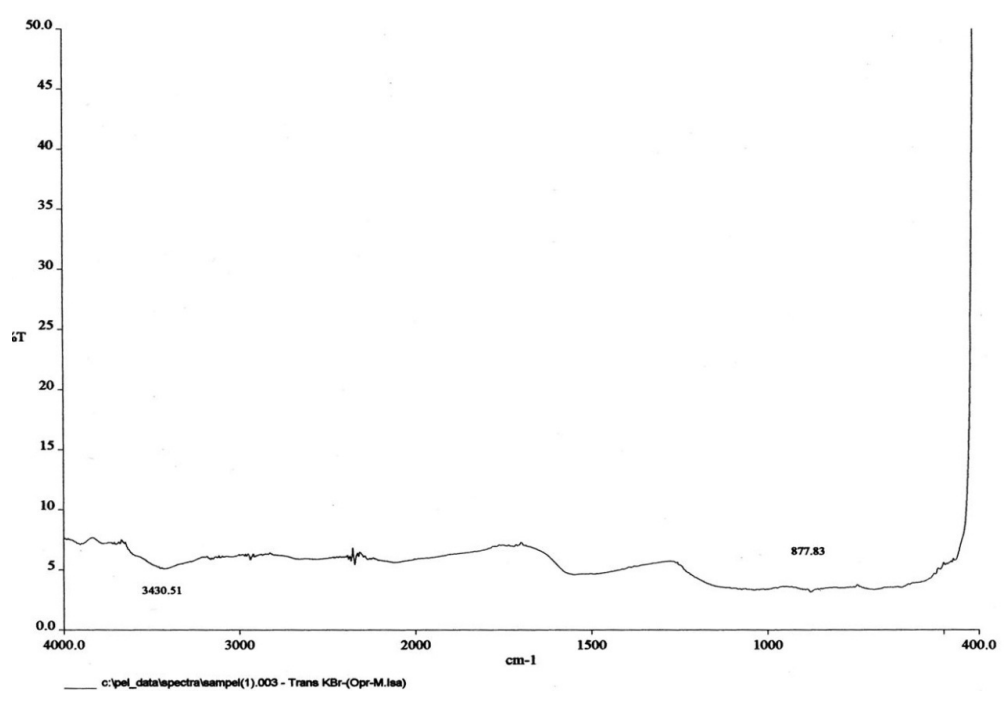

Figure 2. FTIR spectra of MWCNTs (before acid treatment). 


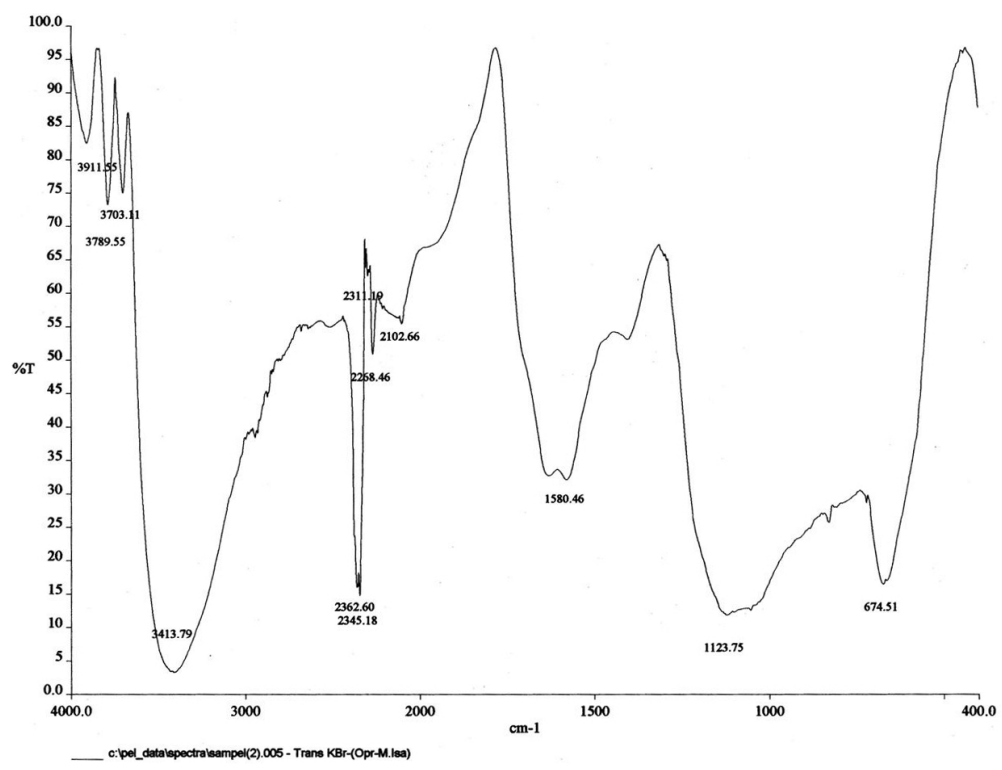

Figure 3. FTIR spectra of MWCNTs (after acid treatment).

\subsection{Transmission Electron Microscopy (TEM)}

TEM microphotographs of pure MWCNTs are shown in Figure 4 (A and B). The figure presents unmodified MWCNTs containing particles with diameters of 5-12 nm. The nanoparticles may be impurities from amorphous carbon and can be removed by acid treatment. According to the supplier, the unmodified MWCNT contains approximately $5 \%$ amorphous carbon. Figure $4 \mathrm{~B}$ demonstrates that most of the nanoparticles were deposited on the surface of the carbon nanotubes and some of them were dispersed throughout the solution used to view the MWCNTs by TEM.
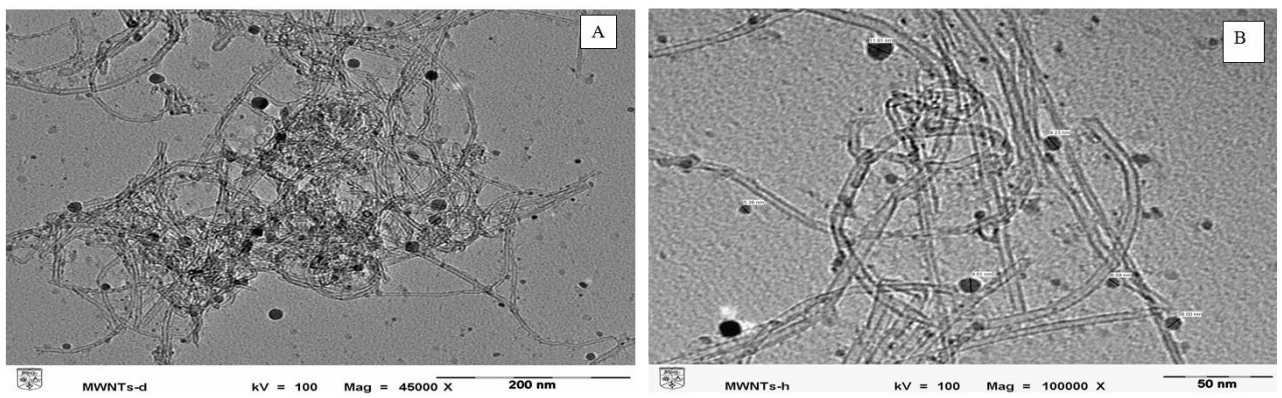

Figure 4. TEM micrograph of Pure MWCNTs before acid treatment with different magnifications (A) 45000 (B) 100000. 
Figure 5 (A and B) displayed no nanoparticles in the acid-modified MWCNTs. The particles might have been removed during acid modification. This reveals that the acid-modified MWCNTs were straight and that some of them aggregated in bundles, which were dispersed well in the matrix. The length of the MWCNTs were reduced during acid modification, since the mixed acid corroded the MWCNTs. TEM microphotographs of the unmodified and acid-modified, curled and entangled MWCNTs demonstrate that the MWCNTs are straight.
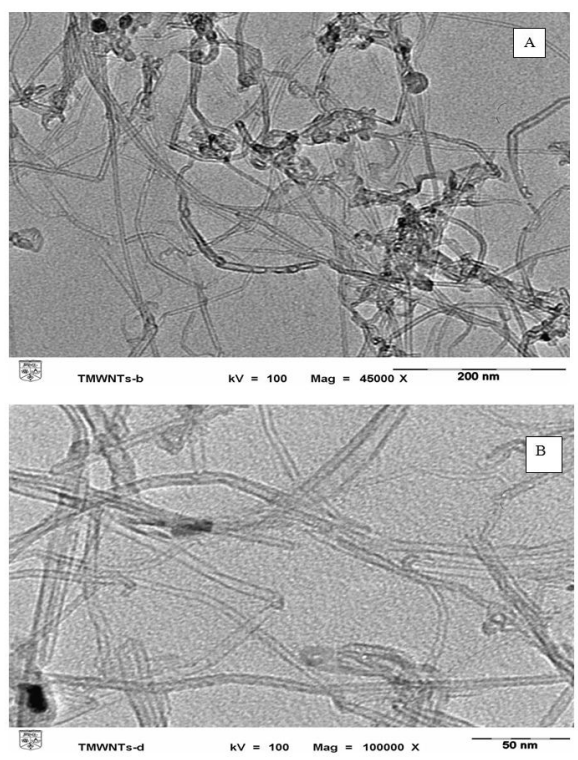

Figure 5. TEM micrograph of MWCNTs after acid treatment with different magnifications (A) 45000 (B) 100000.

\subsection{Mechanical Properties}

\subsubsection{Tensile strength}

The tensile strengths of TPNR reinforced with MWCNTs (with and without treatment) of different percentages $(1 \%, 3 \%, 5 \%$ and $7 \%$ ) are shown in Figure 6. Generally, both MWCNTs exhibited an increasing trend up to $3 \mathrm{wt} \%$ content. Further increments in MWCNTs content decreased the tensile strength compared to the optimum filler loading.

From Figure 6, TPNR with UTMWCNTs and TMWCNTs have optimum results at $3 \mathrm{wt} \%$, which, compared with TPNR, increased by $23 \%$ and $39 \%$, respectively. The tensile strength increased radically as the amount of MWCNTs concentration increased. The mechanical performance, such as tensile properties, strongly depends on several factors such as the properties of the filler reinforcement and matrix, filler content, filler length, filler orientation, 
and processing method and condition. The improvement in the tensile strength may be caused by the good dispersion of MWCNTs in the TPNR matrix, which leads to a strong interaction between the TPNR matrix and MWCNTs. These well-dispersed MWCNTs may have the effect of physically crosslinking points, thus, increasing the tensile strength.

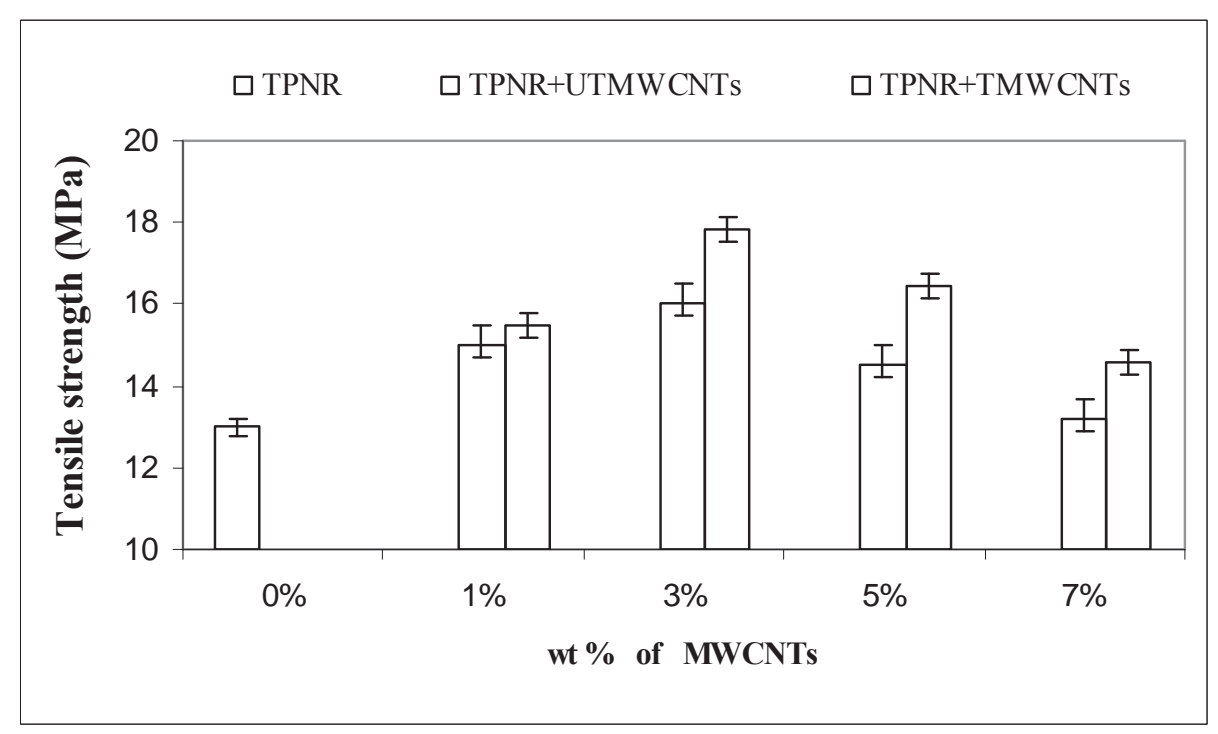

Figure 6. Tensile strength of TPNR reinforced with MWCNTs (with and without treatment).

A good interface between the CNTs and the TPNR is very important for a material to stand the stress. Under load, the matrix distributes the force to the CNTs, which carry most of the applied load. The order of these value is TPNR/TMWCNTs $>$ TPNR/UTMWCNTs $>$ TPNR. The better properties in tensile strength for the TPNR/TMWCNTs nanocomposites could be due to the improved dispersion of the MWCNTs, as well as the response to the opportunities offered by the acid treated MWCNTs. Furthermore, the MWCNTs after acid treatment contain many defects as well as acidic sites on CNTs, such as carboxylic acid, carbonyl and hydroxyl groups. These will greatly enhance the combination of CNTs in a polymer matrix, thus improving the mechanical strength of the nanocomposites [34]. When the content of MWCNTs is higher, the MWCNTs cannot disperse adequately in the TPNR matrix and agglomerate to form a big cluster. This is because of the huge surface energy of MWCNTs as well as the weak interfacial interaction between MWCNTs and TPNR, which leads to inhomogeneous dispersion in the polymer matrix and negative effects on the properties of the resulting composites that cause a decrease in the tensile strength [35]. 


\subsubsection{Young's Modulus}

Figure 7 shows the effect of filler content on the tensile modulus of TPNR reinforced by TMWNTs and UTMWCNTs. The same trend as for the tensile strength in Figure 6 was observed for the tensile modulus of TMWCNTs. Figure 6 clearly shows that the presence of MWCNTs has significantly improved the tensile modulus of the TPNR.

The remarkable increase of Young's modulus with TMWCNTs content shows a greater improvement than that seen in the tensile strength at high content, which indicates that the Young's modulus increases with an increase in the amount of the TMWCNTs. At $3 \mathrm{wt} \%$ of TMWCNTs the Young's modulus is increased by $34 \%$ compared to TPNR. The Young's modulus of UTMWCNTs increased with the increase in the amount of UTMWCNTs. The maximum result was achieved at $3 \mathrm{wt} \%$, with an increase of about $22 \%$, which was due to the good dispersion of nanotubes displaying perfect stress transfer [36].The improvement of modulus is due to the high modulus of MWCNTs [37]. The further addition of TMWCNTs and UTMWCNTs from 5 to $7 \mathrm{wt} \%$ increased the Young modulus dropped respectively.

As explained before, a reduction in performance occurred at higher filler contents for both types of MWCNTs, as depicted in Figure 7. Initially it increases with filler content and then decreases when exceeding the filler loading limit due to the diminishing interfacial fillerpolymer adhesion. It is assumed that aggregates of nanotube ropes effectively reduce the aspect/ratio (length/diameter) of the reinforcement.

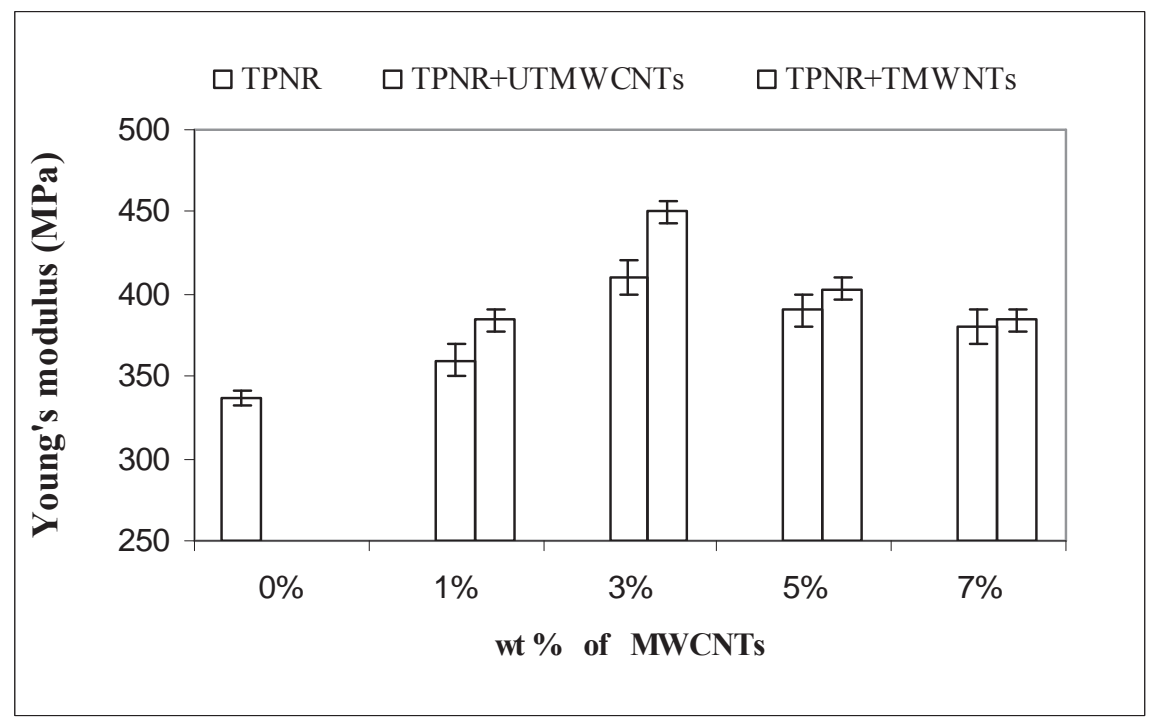

Figure 7. Young's Modulus of TPNR reinforced with MWCNTs (with and without treatment). 


\subsubsection{Elongation at Break}

The elongation at the break of TPNR with TMWCNTs and UTMWCNTs is shown in Figure 8. For TMWCNTs and UTMWCNTs, the elongation at break decreased with the increase in the amount of MWCNTs, compared with TPNR.

It can be deduced that the reinforcing effect of MWCNTs is very marked. As the MWCNTs content in the TPNR increases, the stress level gradually increases, however, the strain of the nanocomposites decreased at the same time. This is because the MWCNTs included in the TPNR matrix behave like physical crosslinking points and restrict the movement of polymer chains. This indicates that, when the amount of CNTs incorporated into the rubber increase it tends to decrease the ductility and the material become stronger and tougher, however, at the same time, it is also

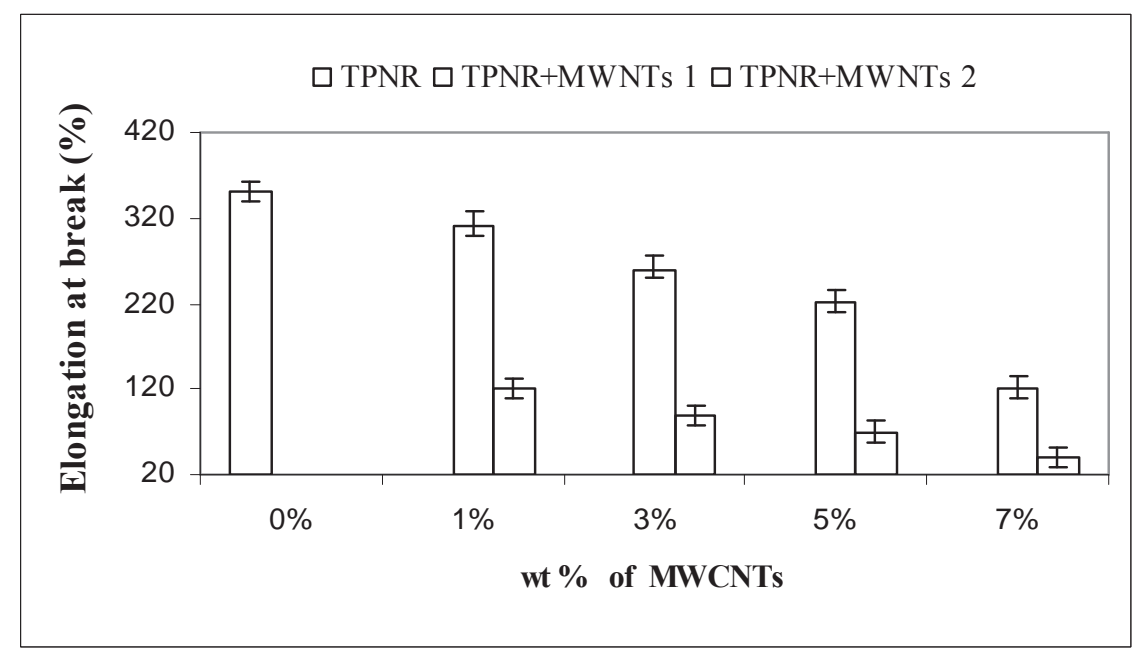

Figure 8. Elongation at Break of TPNR reinforced with MWCNTs (with and without treatment). 


\subsubsection{Impact Strength}

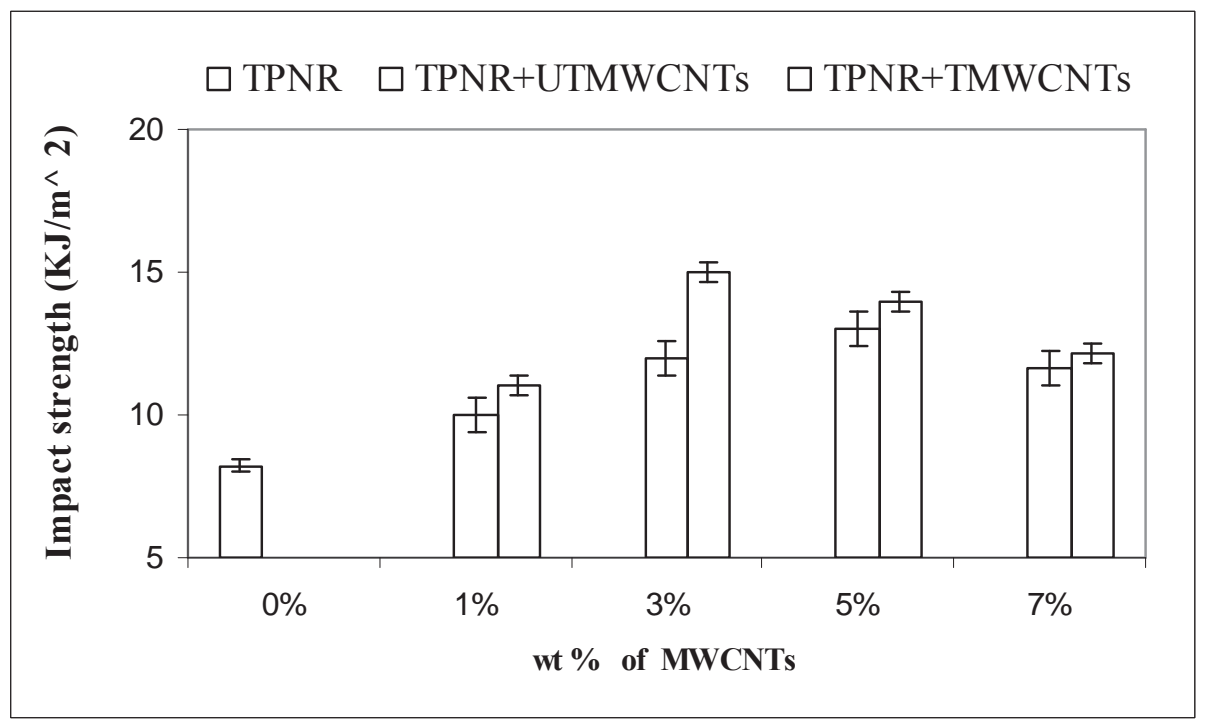

Figure 9. Impact Strength of TPNR reinforced with MWCNTs (with and without treatment).

The effect of filler loading on the impact strength of TPNR/TMWCNTs and TPNR/ UTMWCNTs nanocomposites is given in Figure 9. It shows that incorporation of MWCNTs into TPNR considerably affects the impact strength of TPNR nanocomposites.

The results exhibited better impact strength for TMWCNTs and UTMWCNTs at $3 \mathrm{wt} \%$ with an increase of about $82 \%$ and $46 \%$, respectively. This is due to the better dispersion of carbon nanotubes in the matrix, which generated a significant toughening effect on the TPNR/ TMCWNTs nanocomposite compared with TPNR/UTMWCNTs nanocomposites. However, when the load is transferred to the physical network between the matrix and the filler, the debonding of the chain segments from the filler surface facilitates the relaxation of the matrix entanglement structure, leading to higher impact toughness.

The low impact energy was attributed to the filler content being more than $3 \mathrm{wt} \%$. This will reduce the ability of reinforced composites to absorb energy during fracture propagation. However, in the case of elastomer-toughened polymer, the presence of the elastomer basically produces stress redistribution in the composite, which causes micro cracking or crazing at many sites, thereby resulting in a more efficient energy dissipation mechanism [38].

Consequently, because of their higher surface energy and large aspect ratio, it will be difficult for the nanotubes to disperse in the TPNR when the TMWCNTs and UTMWCNTs content are higher. This will lead to less energy dissipating in the system due to the poor interfacial bonding and induces micro spaces between the filler and polymer matrix. This causes micro-cracks when impact occurs, which induces easy crack propagation. Therefore, 
the higher agglomeration of MWCNTs can cause the mechanical properties of the composites to deteriorate [39].

\subsection{Thermal Properties}

\subsubsection{Glass Transition Temperature}

The dynamic mechanical data shows that the glass transition temperature of the TPNR/ UTMWCNTs and TPNR/TMWCNTs is affected by the addition of the different amounts of MWCNTs, as depicted in Figure 10.

From the figures, the Tg for the TPNR/TMWCNTs nanocomposites is higher than the corresponding temperature for the TPNR and TPNR/UTMWCNTs nanocomposites, usually the $\mathrm{Tg}$ of a polymeric matrix tends to increase with the addition of carbon nanotubes. The rise in $\mathrm{Tg}$ in any polymeric system is associated with a restriction in molecular motion, reduction in free volume and/or a higher degree of crosslinking (TPNR/TMWCNTs > TPNR/ UTMWCNTs) due to the interactions between the polymer chains and the nanoparticles, and the reduction of macromolecular chain mobility.

With the high amount of MWCNTs (after 3wt \%) of TMWCNTs and UTMWCNTs the Tg drops. This might be due to the phase separation/agglomeration of MWCNTs, this allows the macromolecules to move easily. When the content of MWCNTs is higher, the MWCNTs congregate, possibly because the intrinsic van der Waals forces occurs, which leads to bubbles and small aggregates. The conglomerations and matrix holes existing in the network of MWCNTs may perform as defects, which make the macromolecules move easily, and the $\mathrm{Tg}$ of the matrix is decreased. 


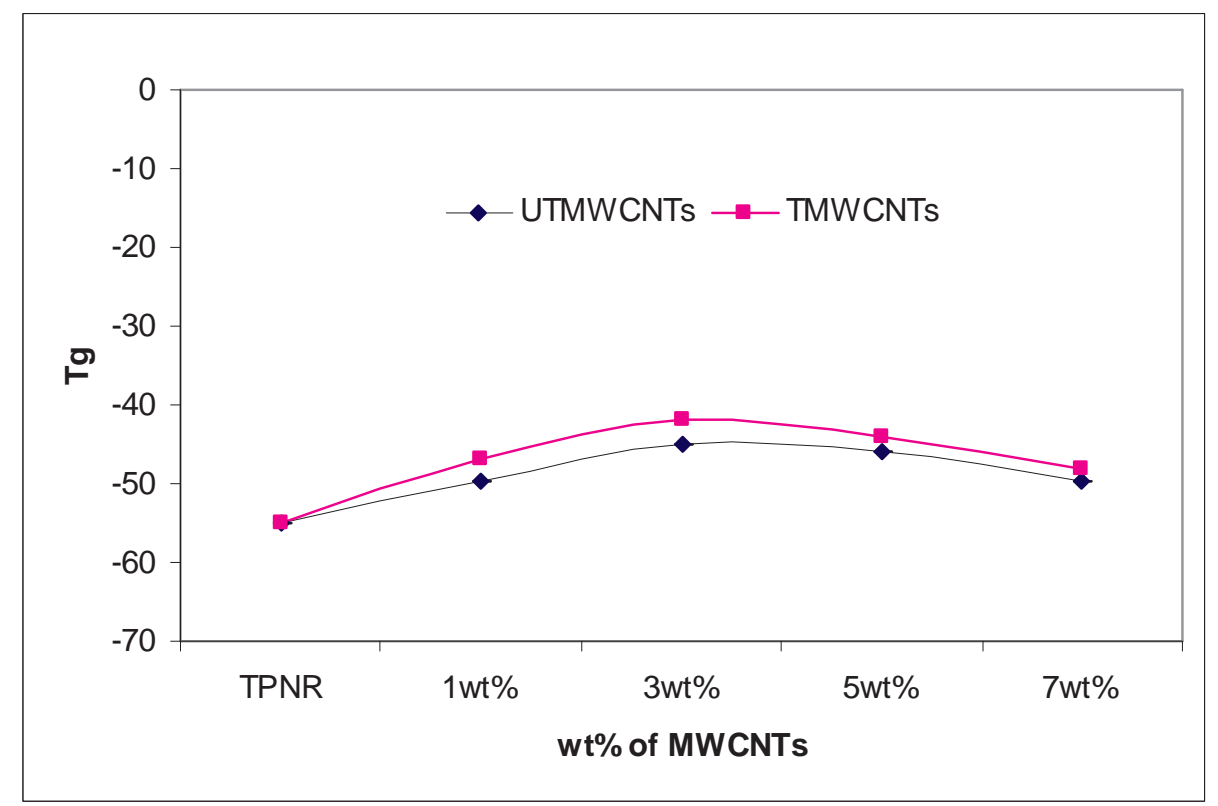

Figure 10. Glass Transition Temperature of TPNR reinforced with MWCNTs (with and without treatment).

\subsubsection{Thermal Conductivity}

To study the effect of MWCNTs filler on thermal conductivity, the temperature was varied from $(30-150){ }^{\circ} \mathrm{C}$. The carbon filler loading was from $1 \mathrm{wt} \%$ to $7 \mathrm{wt} \%$ for two types of carbon nanotubes (UTMWCNTs and TMWCNTs). Introducing MWCNTs to TPNR can significantly enhance the thermal conductivity of the TPNR matrix, as shown in Figure 11 and Figure 12.

As shown in figure 11 at $30^{\circ} \mathrm{C}$ the thermal conductivity of TPNR/TMWCNTs composites, Thermal conductivity increased at $3 w \mathrm{w} \%$ compared to $1 \mathrm{wt} \%, 5 \mathrm{wt} \%$ and $7 \mathrm{wt} \%$, respectively, and for TPNR/UTMWCNTs, the thermal conductivity increased at $3 \mathrm{wt} \%$, as compared to TPNR at the same temperature as shown in figure 12. Thermal transport in the CNT composites includes phonon diffusion in the matrix and ballistic transportation in the filler. 


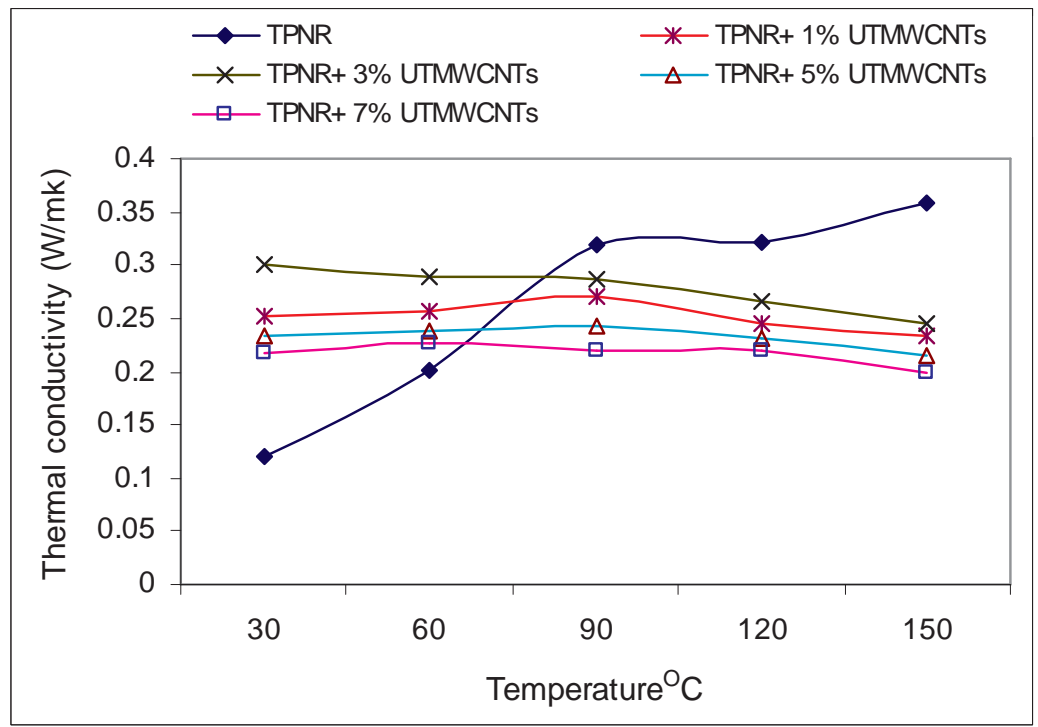

Figure 11. Thermal Conductivity of TPNR reinforced with UTMWCNTS.

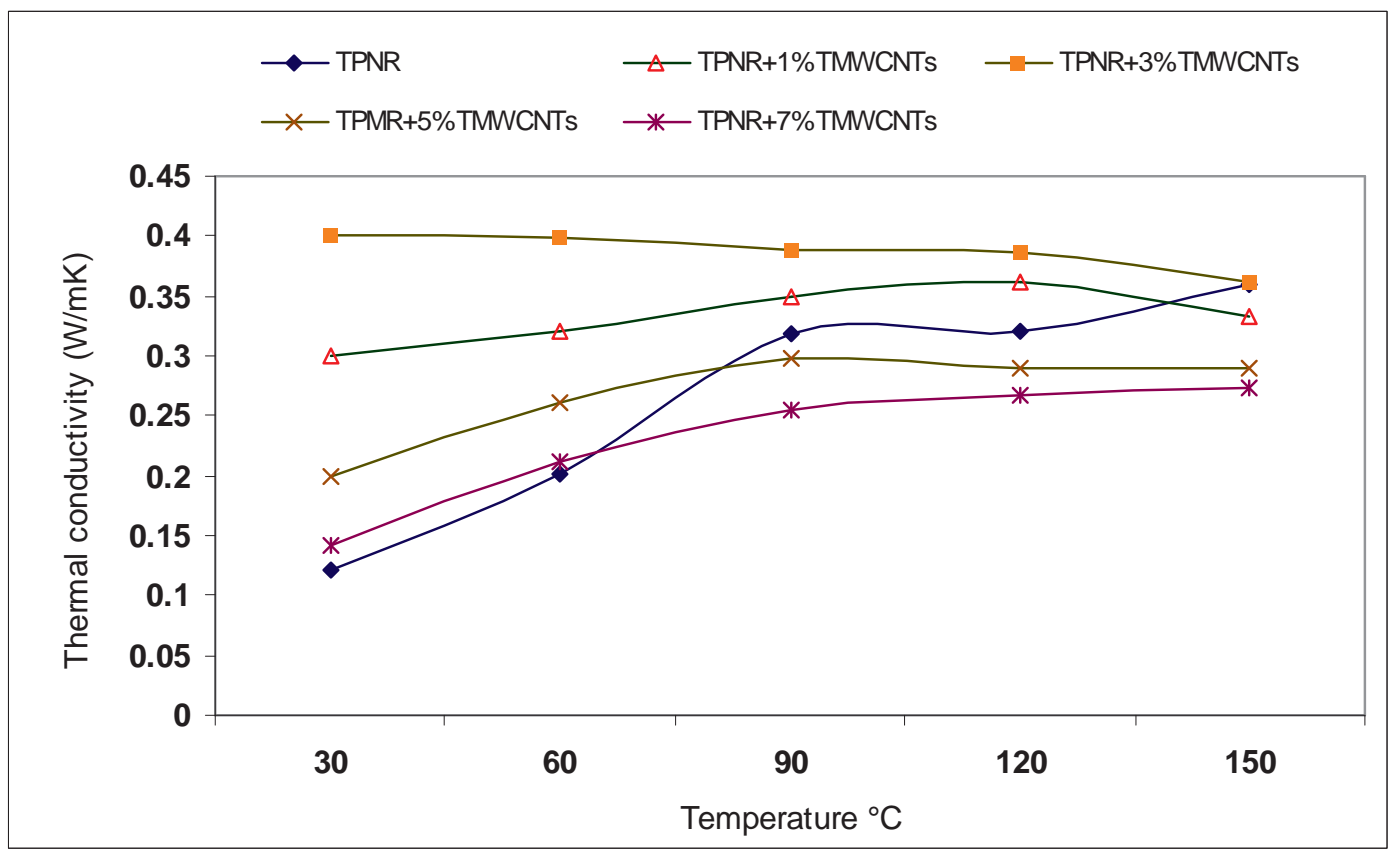

Figure 12. Thermal Conductivity of TPNR reinforced with TMWCNTs. 
The improvement in thermal conductivity in TMWCNTs/TPNR may stem from the improved percolation because of the better dispersion and formation of a network [40]. The dispersion of $1 \mathrm{wt} \%$ and $3 \mathrm{wt} \%$ TMWCNTs is better than $5 \mathrm{wt} \%$ and $7 \mathrm{wt} \%$ in TPNR, at $5 \%$ and $7 \%$ the TMWCNTs agglomerated inside TPNR. Therefore, the large amounts of junctions among the carbon nanotubes form a single conducting path, which is believed to be the reason why the measured thermal conductivity is low. For the UTMWCNTs the conductivity at $3 w t \%$ and $1 w t \%$ is better than $5 w t \%$ and $7 w t \%$, respectively.

The significant enhancement in the thermal conductivity of CNT nanocomposites is possibly attributed to the kinks or twists of UTMWCNTs. When the phonon travels along the nanotubes, if it meets the kinks or twists it would be blocked at those sites. The existence of such kinks or twists in CNTs would lead to a decrease in the effective aspect ratio of the nanotubes [41] when the amount of UTMWCNTs increases, and, thus, the thermal conductivity of UTMWCNTs-TPNR nanocomposites would be reduced. Therefore, the acid treatment of MWCNTs in TPNR could reduce these kinks or twists of TMWCNTs due to the good dispersion of MWCNTs in TPNR, causing the thermal conductivity of the nanocomposites to increase.

Two factors have been proposed to explain the significant enhancement of thermal conductivity with TMWCNTs (1) the rigid linkage between TMWCNTs and TPNR matrix with provides good interface compatibility which may reduce interface thermal resistance; (2) the good interface compatibility allows TMWCNTs to disperse well in the matrix, consequently, the results of the TEM indicate that TMWCNTs possess good dispersion and good compatibility in the TPNR matrix.

The formation of the UTMWCNTs bundles restrict the phonon transport in composites, which maybe be attributed to two reasons (1) the UTMWCNTs aggregation reduces the aspect ratio, consequently, decreasing the contact area between the UTMWCNTs and the TPNR matrix; (2) the UTMWCNTs bundles cause the phenomenon of reciprocal phonon vector, which acts like a heat reservoir and restricts heat flow diffusion.

The resistance to phonon movement from one nanotube to another through the junction will hinder phonon movement and, hence, limit the thermal conductivity. The low thermal conductivity could be partly due to the non-uniform diameter and size, as well as, the defects in and the nano-scale dimension of UTMWNTs. However, the numerous junctions between carbon nanotubes involved in forming a conductive path and the exceptionally low thermal conductance at the interface [42] are believed to be the main reason for the low thermal conductivity.

The effect of reducing the thermal conductivity is the transfer of phonons from nanotube to nanotube. This transition occurs by direct coupling between CNTs, in the case of the improper impregnated ropes, CNT-junctions and agglomerates, or via the matrix. In all these cases, the transition occurs via an interface and, thus, the coupling losses can be attributed to an intense phonon boundary scattering. At the same time the thermal conductivity decreases with the increase in temperature (if the temperature is near the melting point of the matrix). This indicates that the thermal conductivity of the composites is dominated by the 
interface thermal transport between the nanotube/matrix or nanotube/nanotube interface. Thus, it is believed that the decreased effective thermal conductivity of the studied composites could be due to the high interface thermal resistance across the nanotube/matrix or nanotube/nanotube interfaces.

As shown in Figure 11 and Figure 12, the thermal conductivity of TMWCNTs reinforced TPNR matrix composites for all volume fractions studied from $30^{\circ} \mathrm{C}$ to $150^{\circ} \mathrm{C}$ is better than UTMWCNTs. The effect of temperature on the thermal conductivity is clear from $30^{\circ} \mathrm{C}$ to $90^{\circ} \mathrm{C}$, as shown in the figures. This is because of the opposing effect of temperature on the specific heat and thermal diffusivity. Eventually, at high temperatures, as the phonon mean free path is lowered, the thermal conductivity of the matrix approaches the lowest limit and the corresponding thermal resistivity approaches the highest limit.

\subsubsection{Morphological Examination}

The TEM can observe the morphology of UTMWCNTs/TPNR and TPNR/TMWCNTs nanocomposites, which indicates the dispersion abilities of MWCNTs in TPNR matrix before and after treatment of MWCNTs, which summarizes the TEM images of TPNR with $1 \mathrm{wt} \%, 3 \mathrm{wt} \%$ and $7 \mathrm{wt} \%$ UTMWCNTs as shown in figure 12-14. Figure 13 shows the good dispersion of $3 w t \%$ of UTMWCNTs inside TPNR, and exhibits the better interfacial adhesion of UTMWCNTs and TPNR, Figure 14, 7wt\% of UTMWCNTs, shows the poor dispersion and the large UTMWCNTs agglomerates of UTMWCNTs. This is because of the huge surface energy of MWCNTs, as well as, the weak interfacial interaction between UTMWCNTs and TPNR, which leads to inhomogeneous dispersion in the polymer matrix and negative effects on the properties of the resulting composites that causes a decrease in the tensile strength. This supports our results for thermal behavior, which due to the kinks or twists of CNTs can affect the thermal conductivity. Therefore, so when the phonon travels along the nanotube and the phonon meets the kinks or twists, it could be blocked at those sites. The existence of those kinks or twists in CNTs would result in a decrease in the effective aspect ratio of nanotubes at $7 \mathrm{wt} \%$ UTMWCNTs because of agglomeration compared with $3 \mathrm{wt} \%$ of MWCNTs due to the good dispersion. The homogenous dispersion of TMWCNTs in the composites is confirmed by TEM after acid treatment. Figure 15 shows the 3\% TMCWNTs, which are very well dispersed in the matrix, there by suggesting a strong polymer nanotubes interfacial. Strong interfacial adhesion is essential for efficient stress transfer from the matrix to the nanotubes; this supports our observation that the higher efficiency of carbon nanotubes assists in enhancing the properties of TPNR. Low magnification was necessary to observe the poor dispersion of $7 \mathrm{wt} \%$ of TMWCNTs in TPNR as depicted in Figure 17. The figure clearly shows a large number of unbroken carbon nanotubes but less than Figure 14, indicating a poor polymer/nanotube adhesion which is attributed to the reduction in the properties of TPNR/MWCNTs nanocompsites. 


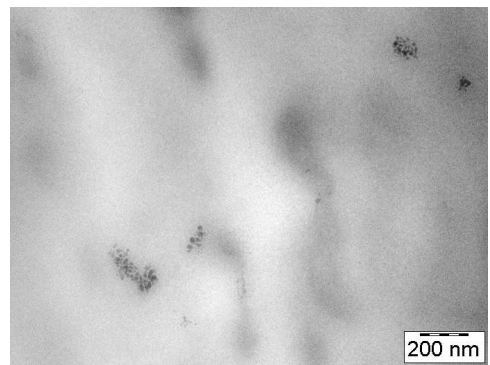

Figure 13. TPNR with $1 \mathrm{wt} \%$ UTMWCNTS.

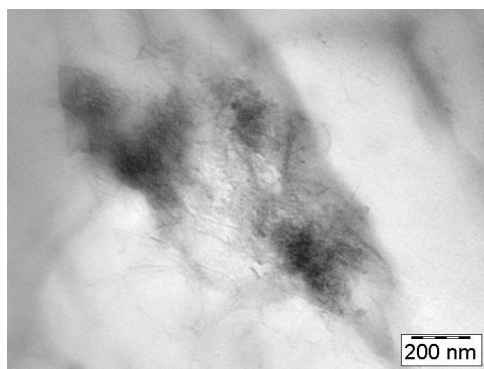

Figure 14. TPNR with $3 w t \%$ UTMWCNTs.

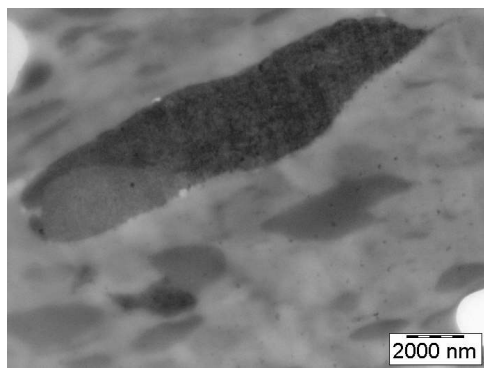

Figure 15. TPNR with 7wt\% UTMWCNTs. 


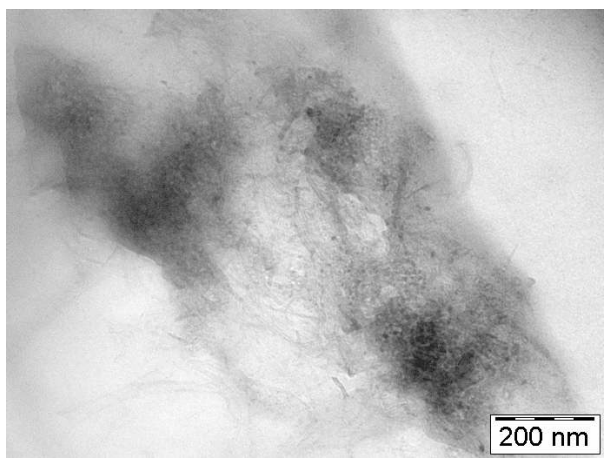

Figure 16. TPNR with $1 w t \%$ TMWCNTS.

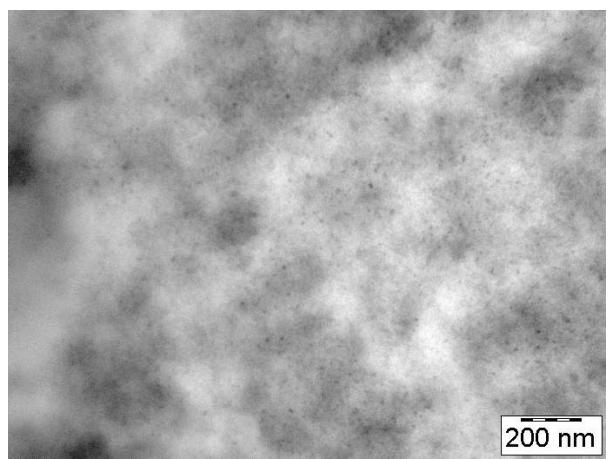

Figure 17. TPNR with $3 w t \%$ TMWCNTs.

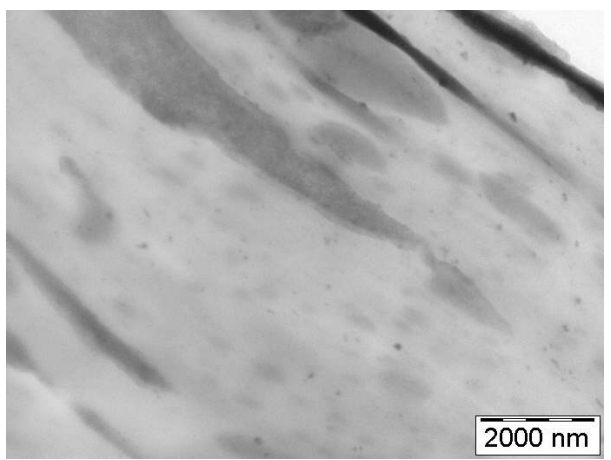

Figure 18. TPNR with 7wt\% TMWCNTs. 


\section{Conclusion}

Recently, it is believed that single-wall carbon nanotubes (SWCNTs), multi-walled carbon nanotubes (MWCNTs), coiled nanotubes and carbon nanofibers (CNFs) can be used as filler in the polymer matrix leading to composites with many enhanced properties, especially in mechanical properties. Furthermore, the inclusion of CNTs in a polymer holds the potential to improve the mechanical, electrical or thermal properties by orders of magnitude well above the performance possible with traditional fillers. In addition many researchers revealed that using functionalized MWCNTs or surface modification of MWCNTs as filler enhanced the properties of nanocomposites. This enhancement was probably suggested because of the homogenous dispersion and stronger interaction between the MWCNTs and the polymer matrix. After being treated with an acid, some functional groups were introduced onto the MWCNTs surface, which can form a physical interaction with the polymer chain. In this chapter, the effect of multi-walled carbon nanotubes with and without acid treatment on the properties of thermoplastic natural rubber (TPNR) was investigated. Two types of MWCNTs were introduced into TPNR, which are untreated UTMWCNTs (without acid treatment) and treated TMWCNTs (with acid treated MWCNTs). The acid treatment of MWCNTs removed catalytic impurities and generated functional groups such as hydroxyl, carbonyl and mainly carboxylic acid.

The results in this chapter show that the properties of MWCNTs can be improved by using this method. The TEM micrograph has shown that the effect of acid treatment has roughened the MWCNTs surface and also reduced the agglomeration. Various functional groups have been confirmed using FTIR. The TPNR nanocomposite was prepared using the melt blending method. MWCNTs are incorporated in the TPNR nanocomposite at different compositions which is 1, 3, 5 and $7 \mathrm{wt} \%$. The addition of MWCNTs in the TPNR matrix improved the mechanical properties. At $3 \mathrm{wt} \%$, the tensile strength and Young's modulus of TPNR/UTMWCNTs increased 23\% and 22\%, respectively. For TPNR/TMWCNTs the optimum result of tensile strength and Young's modulus was recorded at 3\% which increased $39 \%$ and $34 \%$, respectively. In the addition the elongation of break decreased by increasing the amount of both types of MWCNTs.

The results exhibited better impact strength for UTMWCNT and TMWCNT at $3 \mathrm{wt} \%$ with an increase of almost $46 \%$ and $82 \%$, respectively. The reinforcing effect of two types of MWCNTs was also confirmed by dynamic mechanical analysis where the addition of MWCNTs have increased in the glass transition temperature (Tg) with an increase in the amount of MWCNTs (optimum at 3wt \%) and it increased with the TMWCNTs more than the UTMWCNTs. Thermal conductivity improved with TMWCNTs compared to the UTMWCNTs. The homogeneous dispersion of two types of the MWNTs throughout the TPNR matrix and strong interfacial adhesion between the MWCNTs and the matrix as confirmed by the TEM images are proposed to be responsible for the significant mechanical enhancement. 


\section{Acknowledgements}

The authors would like to thank the Malaysian Government and Universiti Kebangsaan Malaysia (UKM) under Science Fund Grant UKM-OUP-NBT-29-142/2011 and UKMOUP-2012-135 for financial support.

\section{Author details}

Mou'ad A. Tarawneh* and Sahrim Hj. Ahmad

*Address all correspondence to: moaath20042002@yahoo.com

School of Applied Physics, Faculty of Science and Technology, Universiti Kebangsaan Malaysia, Malaysia

\section{References}

[1] Sakurada, I. (1985). Polyvinyl Alcohol Fibres. International Fibre Science and Technology Series, 6, Marcel Dekker, Inc., New York.

[2] Li, C. Y., \& Chou, T. W. (2003). A structural mechanics approach for the analysis of carbon nanotubes. Int. J. Solids Struct., 40, 2487-2499.

[3] Hernandez, E., Goze, C., Bernier, P., \& Rubio, A. (1998). Elastic Properties of C and BxCyNz Composite Nanotubes. Phys. Rev. Lett., 80, 4502-4505.

[4] Yakobson, B. I., Campbell, M. P., Brabec, C. J., \& Bernholc, J. (1997). High strain rate fracture and C-chain unraveling in carbon nanotubes. Comp. Mater. Sci, 8, 341-348.

[5] Demcyzk, B. G., Wang, Y. M., Cumings, J., Hetman, M., Han, W., Zettl, A., \& Ritchie, R. O. (2002). Direct mechanical measurement of the tensile strength and elastic modulus of multiwalled carbon nanotubes. Mater. Sci. Eng. A, 334, 173-178.

[6] Qian, E. C., Dickey, , Andrews, R., \& Rantell, T. (2000). Load transfer and deformation mechanisms in carbon nanotube-polystyrene composites. Appl. Phys. Lett, 76, 2868-2870.

[7] Lopez, M. A., Valentine, L., Biagiotti, J., \& Kenny, J. M. (2005). Thermal and mechanical properties of single-walled carbon nanotubes-polypropylene composites prepared by melt processing. Carbon, 43, 1499-1505.

[8] Liu, T., Phang, I. Y., Shen, L., Chow, S. Y., \& Zhang, W. D. (2004). Morphology and mechanical properties of multi-walled carbon nanotubes reinforced nylon-6 nanocomposites. Macromolecules, 37, 7214-7222. 
[9] Frogley, M. D., Ravich, D., \& Wagner, H. D. (2003). Mechanical properties of carbon nanoparticle-reinforced elastomers. Compos. Sci. Technol, 63, 1647-1654.

[10] Koerner, H., Liu, W., Alexander, M., Mirau, P., Dowty, H., \& Vaia, R. A. (2005). Deformation-morphology correlations in electrically conductive carbon nanotube- Thermoplastic polyurethane nanocomposites. Polymer, 46, 4405-4420.

[11] Berber, S., Kwon, Y. K., \& Tomanck, D. (2000). Unusually High Thermal Conductivity of Carbon Nanotubes. Phys. Rev. Lett, 84, 4613-4616.

[12] Biercuk, M. J., Llaguno, M. C., Radosavljevic, M., Hyun, J. K., \& Johnson, A. T. (2002). Carbon nanotube composites for thermal management. Appl. Phys. Lett, 80, 2767-2769.

[13] Hone, J. W., Piskoti, C., \& Zettl, A. (1999). Thermal conductivity of single-walled carbon nanotubes. Phys. Rev. B, 59, 2514-2516.

[14] Gonnet, P., Liang, Z., Choi, E. S., Kadambala, R. S., Zhang, C., Brooks, J. S., Wang, B., \& Kramer, L. (2006). Thermal conductivity of magnetically aligned carbon nanotube buckypapers and nanocomposites. Current Applied Physics, 6, 119-122.

[15] Fangming, D., Csaba, G., Takashi, K., John, E. F., \& Karen, I. W. (2006). An infiltration method for preparing single-wall nanotube/epoxy composites with improved thermal conductivity. J. Poly. Sci. Part B: Poly. Phys, 44, 1513-1519.

[16] Sinha, S., Barjami, Iannacchione. G., Schwab, A., \& Muench, G. (2005). Off-axis Thermal Properties of Carbon Nanotube Films. J. Nanoparticle Res, 7, 651-657.

[17] Wen-Tai, H., \& Nyan-Hwa, T. (2008). Investigations on the thermal conductivity of composites reinforced with carbon nanotubes. Diamond \& Related Materials, 17, 1577-1581.

[18] Moisala, Q. L., Kinloch, I. A., \& Windle, A. H. (2006). Thermal and electrical conductivity of single- and multi-walled carbon nanotube-epoxy composites. Composites Science and Technology, 66, 1285-1288.

[19] Shiren, W., Richard, L., Ben, W., \& Chuck, Z. (2009). Dispersion and thermal conductivity of carbon nanotube composites. Carbon, 47, 53-57.

[20] Assael, M. J., Antoniadis, K. D., \& Tzetzis, D. (2008). The use of the transient hot-wire technique for measurement of the thermal conductivity of an epoxy-resin reinforced with glass fibres and/or carbon multi-walled nanotubes. Composites Science and Technology, 68, 3178-3183.

[21] Oconnell, M. J., Boul, P., Ericson, L. M., Huffman, C., Wang, Y., Haroz, E., Kuper, C., Tour, J., Ausman, K. D., \& Smalley, R. D. (2001). Reversible water-solublization of single-walled carbon nanotubes by polymer wrapping. Chem Phys Lett, 342, 265-271.

[22] Niyogi, S., Hamon, M. A., Perea, D. E., Kang, C. B., Zhao, B., \& Pal, S. K. (2003). U1trasonic dispersion of single-walled carbon nanotubes. J. Phys. Chem. B, 107, 8799-8812. 
[23] Viswanathan, G., Chakrapani, N., Yang, H., Wei, B., Chung, H., \& Cho, K. (2003). Single-step in situ synthesis of polymer-grafted single-wallednanotube composites. J Am Chem Soc, 125, 9258-9259.

[24] Xie, X. L., Mai, Y. W., \& Zhou, X. P. (2005). Dispersion and alignment of carbon nanotubes in polymer matrix: A review. Mater Sci Eng, 49, 89-112.

[25] Wang, Y., Wu, J., \& Wei, F. (2003). A treatment method to give separated multiwalled carbon nanotubes with high purity, high crystallization and a large aspect ratio. Carbon, 41, 2939-2948.

[26] Abdullah, I., \& Dahlan, M. (1998). Thermoplastic natural rubber blends. Prog. Polym. Sci, 23, 665-706.

[27] Abdullah, I., \& Ahmad, S. (1992). Liquid NR as a compatibilizer in the blending of NR with PP. Mater. Forum, 16, 353-357.

[28] Tarawneh, Mou'ad A., Ahmad, Sahrim Hj., Rasid, Rozaidi, Yahya, S. Y., \& Eh Noum, Se Yong. (2011). Thermal Behavior of a MWNT Reinforced Thermoplastic Natural Rubber Nanocomposite. Journal of Reinforced Plastic and Composites, 30(3), 216-221.

[29] Tarawneh, Mou'ad A., Ahmad, Sahrim Hj., Rasid, Rozaidi, Yahya, S. Y., \& Eh Noum, Se Yong. (2011). Enhancement of the Mechanical Properties of Thermoplastic Natural Rubber Using Multi-walled Carbon Nanotubes. Journal of Reinforced Plastic and Composites, 30(4), 363-368.

[30] Tarawneh, Mou'ad A., \& Ahmad, Sahrim Hj. Reinforced Thermoplastic Natural Rubber (TPNR) Composites with Different Types of Carbon Nanotubes (MWNTS), Book Name Carbon Nanotubes - Synthesis, Characterization, Applications (Chapter 21, Page 443-468). InTech. July 2011, 978-9-53307-497-9.

[31] Chang-Eui, H., Joong-Hee, L., Prashantha, K., \& Suresh, G. A. (2007). Composites Science and Technology, 67, 1027-1034.

[32] Seung, H. L., Eunnari, C., So, H. J., \& Jae, R. Y. (2007). Rheological and electrical properties of polypropylene composites containing functionalized multi-walled carbon nanotubes and compatibilizers. Carbon, 45, 2810-2822.

[33] Hirsch, A. (2002). Functionalization of single-walled carbon nanotubes. Angew Chem Int Ed, 41, 1853-1859.

[34] Sang, H. J., Young-Bin, P., \& Kwan, H. Y. (2007). Rheological and mechanical properties of surface modified multi-walled carbon nanotube-filled PET composite. Composites Science and Technology, 67, 3434-3441.

[35] Potschke, P., Fornes, T. D., \& Paul, D. R. (2002). Rheological behavior of multi-walled carbon nanotubes/polycarbonate composites. Polymer, 43, 3247-3255.

[36] Treacy, T. W. E., \& Gibson, J. M. (1996). Exceptionally high Young's modulus observed for individual carbon nanotubes. Nature, 381, 678-680. 
[37] Canche-Escamilla, G., Rodriguez-Laviada, J., Cauich-Cupul, J. I., Mendizabal, E., Puig, J. E., \& Herrera-Franco, P. J. (2002). Flexural, impact and compressive properties of a rigid-thermoplastic matrix/cellulose fiber reinforced composites. Compos. Part (A): Appl. Sci. E Manufact., 33, 539-549.

[38] Jianfeng, S., Weishi, H., Liping, W., Yizhe, H., \& Mingxin, Y. (2007). The reinforcement role of different amino-functionalized multi-walled carbon nanotubes in epoxy nanocomposites. Composites Science and Technology, 67, 3041-3050.

[39] Kumar, S., Alam, M. A., \& Murthy, J. Y. (2007). Effect of percolation on thermal transport in nanotube composites. Appl Phys Lett, 90, 104105-1-104105-3.

[40] Nan, C. W., Shi, Z., \& Lin, Y. (2003). A simple model for thermal conductivity of carbon nanotube-based composites. Chem Phys Lett., 375, 666-669.

[41] Yunsheng, X., Gunawidjaja, R., \& Beckry, Abdel-Magid. (2006). Thermal behavior of single-walled carbon nanotube polymer-matrix composites. Composites A, 37(1), 114-121.

[42] Ramasamy, S., Shuqi, G., Toshiyuki, N., \& Yutaka, K. (2007). Thermal conductivity in multi-walled carbon nanotubes/silica-based nanocomposites. Scripta Materialia, 56, 265-268. 
